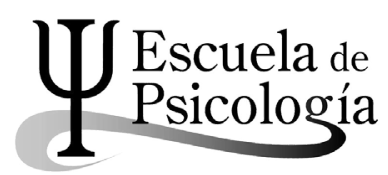

Wímb lu, Rev. electrónica de estudiantes Esc. de psicología, Univ. de Costa Rica. 9 (2): 23-43, 2014 / ISSN: 1659-2107

\title{
ALGUNOS ESTEREOTIPOS, ROLES Y REFRANES SOBRE GÉNERO VIGENTES EN LA ACTUALIDAD EN ESTUDIANTES DE LA UNIVERSIDAD DE COSTA RICA
}

\author{
Some stereotypes, roles and sayings related to gender \\ current for students at University of Costa Rica
}

Doris Céspedes*

\begin{abstract}
Resumen: Este artículo consiste en una investigación de tipo decriptiva con 505 estudiantes de la Universidad de Costa Rica, a los cuales se les aplicó una encuesta durante el mes de junio del año 2013 para conocer si algunos estereotipos y roles de género siguen vigentes en el contexto universitario en el siglo XXI. En este estudio se pudo encontrar que los estereotipos y roles de género no están tan presentes en la cotidianidad de las aulas universitarias, ya sea en hombres como en mujeres; los resultados muestran que se han generado cambios en la percepción de algunos de los estereotipos y de los roles, sin embargo, en cuanto a los refranes no se nota mayor cambio.

Los resultados no señalan una diferencia amplia en relación a los porcentajes entre la edad, sexo, lugar de procedencia, carrera o área de estudio de los y las participantes en la encuesta en cuanto a sus respuestas.

Concretamente, en donde se dan más diferencias es en los porcentajes de respuestas sobre el ítem de la afectividad de las mujeres a través de la expresión sentimental, y en la percepción que se sigue teniendo de una relación amorosa entre un hombre mayor y una mujer joven, lo que hace evidente la vigencia de ciertos estereotipos.

Y finalmente, puede observarse en los resultados que la vigencia de los refranes se mantiene en los y las jóvenes universitarias de la actualidad.
\end{abstract}

Palabras claves: estereotipos, roles, refranes, género.

Universidad de Costa Rica, Escuela de psicología, Costa Rica. Correo: doriscespedes10@ hotmail.com

Recepción: 9/10/2013 Aceptación: 10/5/2014 


\begin{abstract}
This article was based on results from a descriptive research with 505 participants, all students enrolled at Universidad de Costa Rica, who were interviewed during the month of July, 2013; the role was to determine whether, still in current times, certain gender roles and stereotypes exist in the college's context. It was found that those roles and stereotypes are not considerably present in the daily lifes of neither male nor female students in their classes. The results tend to indicate that changes in perception have taken place; however, popular sayings are still used by the young student population. Results don't show a significant difference in responses in relation to the participant's age, gender, location, degree or field of study.

There were some concrete differences in the participant's responses, particularly in the entry related to woman's emotional expression, and the perceptions that still remain regarding a romantic relationship between and older man and a young woman, which still provide some margin of validity to those stereotypes.
\end{abstract}

Key Words: roles, stereotypes, gender, sayings.

\title{
Introducción
}

El presente artículo tiene como objetivo reflexionar acerca de la percepción, legitimización y mantenimiento de algunos estereotipos, roles de género y refranes, debido a que al preguntar en las aulas a los y las estudiantes sobre algunos de ellos, dan respuestas afirmativas de haberlos escuchado. Por lo anterior, describir si aún en el actual siglo XXI y en pleno año 2013, siguen vigentes algunos de los estereotipos y roles de género en estudiantes de la Universidad de Costa Rica originó los cuestionamientos que dan el insumo de este documento.

De acuerdo a Chaves (2005), que refiere a Díaz (1999), el género es una construcción socio-cultural que asigna determinados comportamientos a hombres y a mujeres, y los diferencia en términos de papeles y actividades que desarrollan en la sociedad, esta diferenciación que establece lo femenino y lo masculino, a la vez, va fortaleciendo jerarquías, entre unos y otras, es decir va estableciendo relaciones de poder y situaciones de inequidad entre ellos y ellas, que como consecuencia crea muchas veces la violencia entre géneros.

Los estereotipos y roles de género en la actualidad suponen un cambio de percepciones y de acciones en las nuevas generaciones, esto desde lo teórico, sin embargo desde la cotidianidad y desde la práctica, parece que se siguen manteniendo las formas tradicionales a través de una herencia oculta por lo menos en los y las estudiantes universitarias, aunque en las discusiones en clases, las y los jóvenes opinan que esto ha variado aunque sea un poco. Luego de analizar algunos roles que todavía se mantienen, como las responsabilidades del orden, aseo y arte culinario, sobre todo se dejan decir algunas de estas estudiantes en la clase, que cuando hay una hermana en la familia, se tiende a responsabilizarla de la atención de sus hermanos varones.

La razón que origina este estudio, se debe a la preocupación e inquietud de la autora como docente universitaria, a partir de la experiencia por varios años de contar con la colaboración del MSc. José Manuel Salas como invitado a las clases. 
Él ofrece una charla en el tema de género, específicamente sobre Masculinidad, en la cual aprovecha el espacio para introducir varias preguntas a manera de encuesta verbal sobre frases o refranes que se han utilizado desde hace años. Esta actividad dio pie a la inquietud y de esta manera a la creación de una encuesta de opinión de tipo descriptiva para elaborar el presente artículo, contando con la colaboración de estudiantes de los cursos de Psicología General de la Escuela de Psicología, para su aplicación en el campus universitario y así indagar sobre algunas percepciones de los estereotipos y roles vigentes en los y las estudiantes universitarios del presente año 2013, encuesta aplicada a estudiantes de diferentes niveles académicos y diversas carreras de la Universidad de Costa Rica.

Como docente de psicología es impactante consultar en la época actual a los y las estudiantes sobre algunos estereotipos, roles, refranes que se han escuchado por generaciones, llamando la atención que en generaciones tan distintas a las de hace bastantes décadas y en la década actual, por lo menos algunos refranes continúan presentes en forma manifiesta, lo que induce a pensar que se siguen legitimando esos estereotipos y roles de género, inevitablemente a través del inconsciente colectivo, como lo mencionó Carl Gustav Jung (1970), definiendo a este tipo de inconsciente, como la existencia de un sustrato común a los seres humanos de todos los tiempos y lugares del mundo, constituido por símbolos primitivos con los que se expresa un contenido de la psique que está más allá de la razón. Aunque la anterior argumentación supone la dificultad de eliminar estereotipos afianzados históricamente, y ha sido imposible hacerlo casi en su totalidad, se hace obligatorio continuar la lucha para que se reduzcan, siquiera esa es la expectativa.

Por otra parte, es imprescindible, según Camacho y Watson (2008), que en la construcción de una sociedad basada en la igualdad de oportunidades y derechos entre hombres y mujeres, es absolutamente necesario e indispensable generar cambios culturales desde las edades más tempranas, sin embargo parece no estar ocurriendo en nuestro contexto social, porque escuchando las noticias en el diario vivir, con frecuencia se sigue informando de hechos de violencia contra la mujer, hechos que se sabe muchas de las veces son fundamentados porque el hombre siente que está perdiendo el poder. Y si tomamos en cuenta que la identidad masculina se caracteriza por la reafirmación permanente de su poder, entonces podrán los lectores observar en los resultados de la encuesta aplicada, que aunque las respuestas mayoritariamente brindan una percepción contraria a la práctica, lo que más llama la atención es que ocurra en la población estudiantil de la Universidad de Costa Rica, que es de suponer tiene una amplia instrucción académica.

Por supuesto, es innegable que el tema discutido en este artículo se genera mediante procesos históricos, sociales, culturales y educativos, en donde 
lastimosamente en los diferentes contextos se promueven las creencias sociales sobre la consagración de roles en función del sexo, y sobre la base de espacios que han sido establecidos y valorados de manera desigual, lo que pareciera seguir siendo conveniente para unos más que para otros u otras, y así mantener muchas de las veces la discriminación e inequidad de género, sin dejar de señalar que obedece a necesidades de orden económico, político e ideológico propias del surgimiento del patriarcado, lo que ha permitido ejercer un mayor control sobre la vida de las personas de manera desigual.

Pero precisamente, cuando en los diferentes espacios de tertulia, conversación o reflexión en pasillos o aulas universitarias, se escucha a los y las jóvenes actuando verbal y físicamente con estereotipos o roles que por generaciones no han cambiado, es cuando se hace obligatorio reflexionar sobre la legitimación de estos aprendizajes y pensar en la subsistencia de un currículo oculto que sigue de alguna manera posibilitando el tardío cambio de actitudes que menoscaban la integridad de hombres y mujeres, lo que afecta no solo su condición física, sino también la salud mental.

Espero que a través de este artículo aflore la reflexión para comprender como se reproduce y legitima por medio de los estereotipos, roles y refranes la discriminación e inequidad sin a veces percatarnos de que es por medio de la utilización de un lenguaje implícito reproducido a veces inconscientemente, pero que al día de hoy se reproducen arquetipos que favorecen los mecanismos de dominación en esa hegemonía que le es conveniente a ciertos sectores de la sociedad. Deseo que la lectura que se brinda aquí pueda ayudar a no solo concientizar sino que con esta se puedan emprender acciones, ya sea individual o colectivamente, porque los prejuicios de género los tenemos todos, y de alguna manera nos condicionan y es importante poder modificar esas ideas preconcebidas, que en última instancia no hacen más que un sesgo a nuestra realidad.

A continuación se definen conceptualmente algunos términos que dan la fundamentación teórica a esta reflexión, siguiendo con la metodología, para agregar el análisis sobre los resultados más importantes de la encuesta que sirvieron para las conclusiones y la creación de este artículo.

\section{Marco Teórico}

En este apartado es importante definir conceptos como estereotipo, rol de género, percepción y percepción social y algunos tópicos de las teorías de género, que son los constructos teóricos del objeto de estudio de este artículo, así por ejemplo el concepto de estereotipo en primera instancia ciertamente presenta una larga historia y poco consenso, en tantas de las definiciones propuestas.

En su historia el término estereotipo, su etimología ha sido compleja, de esta manera su definición es diferente no solo interdisciplinariamente, sino que en 
diferentes contextos como por ejemplo el de las ciencias sociales. Sin embargo, es a finales del siglo XVIII, que nace en el mundo de la imprenta la palabra estereotipo, como lo menciona Eguizábal, R. (2002), el término estereotipo se originó en el mundo de las artes gráficas, para designar las impresiones salidas de un molde, por lo que de acuerdo al autor, el término fue aplicable a todo lo que parece "salido de un molde, ya hecho, invariable", y fue la psiquiatría como ciencia la primera en adoptarlo, para designar aquella persistente reiteración de una conducta propia de ciertas patologías mentales.

De acuerdo a Cano (1993), menciona que fueron Katz y Braly los que desarrollaron una nueva técnica de medición de estereotipos a través de una lista de adjetivos, para averiguar qué adjetivos relativos a rasgos de la personalidad eran los más propios de cada grupo étnico, racial o nacional, definiendo estereotipo como "una impresión fijada, que se corresponde muy poco con los datos que tiende a representar, y resulta de que definimos primero y observamos en segundo lugar".

Sin embargo con la evolución de este término más adelante, Allport (1971) aportó una nueva definición de estereotipo, vinculada al término de prejuicio, definiéndolo como "una creencia exagerada asociada a una categoría". Para este autor, los prejuicios son una decisión categorial y los estereotipos se corresponden con los contenidos de las categorías irracionales, o ideas fijas que acompañan a éstas, de forma precisa para él, el estereotipo pasa a ser entendido como el componente cognitivo del prejuicio.

Para Eguizábal (2002), existen diferentes conceptualizaciones en la historia de la definición del estereotipo social: la corriente patológica que lo considera un mecanismo negativo de la percepción; otra conceptualización centrada en el interés por el componente evaluativo más que la negatividad del estereotipo; y la perspectiva cognitiva que explica el estereotipo como un proceso natural de la percepción.

Por último agrega Eguizábal (2002), que existieron diversos teóricos, y menciona a Walter Lippman como el que hizo surgir esta corriente con su Public Opinion en el año 1922, llevando a cabo un análisis conceptual en el que consideraba al estereotipo como una construcción individual y social de la percepción sujeta a distorsiones tanto psicológicas como socio-ambientales determinadas por la economía cognitiva a la que se debían.

Finalmente para efectos de este estudio, por su profundidad, se utiliza la propuesta que hace Herrero (2006), que menciona las funciones «constructivas» de los estereotipos señalando que son esquemas ampliamente compartidos en el mundo social (aunque puedan variar de unos ambientes a otros), esquemas que son preconstruidos (el individuo los recibe de la lengua o del ambiente sociocultural) y contribuyen a organizar las representaciones colectivas, las opiniones intercambiadas y el imaginario social (conceptos, nociones, mentalidades, 
prejuicios, valores, etc.), además agrega que los estereotipos de pensamiento se apoyan con frecuencia en los estereotipos lingüísticos o fraseológicos recurriendo a sintagmas o a enunciados que adoptan una forma verbal fija para comunicar un mensaje abstracto o conceptual global (locuciones de diversos tipos, refranes y dichos populares, enunciados conversacionales estereotipados rutinarios, fórmulas rituales de cortesía, lemas, consignas, etc.), para concluir que esas fórmulas son recurrentes y se repiten en múltiples situaciones.

La anterior definición se refiere al estereotipo como definición general, por lo que es necesario la definición de estereotipo de género, para la cual existen múltiples definiciones, una de estas es la de Parga (2008), que define los estereotipos de género como el conjunto de reglas (explicitas e implícitas), normas, ritos y rutinas, que forman parte de las estructuras, del conjunto de prácticas organizativas en los diferentes campos sociales.

Un problema que no puede obviarse, es la función que finalmente tienen los estereotipos y roles, que acarrean muchas consecuencia negativas, como lo menciona González (2000), las situaciones de explotación en la sociedad son el resultado de situaciones históricas relacionadas con una estructura socioeconómica, en donde la trasmisión ideológica es un factor fundamental para mantener la dominación que desfavorece a unos sobre otros, específicamente en el caso de las mujeres sufren más estos embates, produciendo relaciones entre hombres y mujeres de inequidad y discriminación, ejemplo de esto es la división del trabajo, en donde los roles evidencian desventajas abismales.

Por otra parte, para hacer referencia a la definición de rol, de acuerdo a Alfonso y Aguado (S.F.) son aquellos papeles, personajes o rótulos que especifican nuestras supuestas obligaciones y expectativas y se construyen siempre en función del entorno, en definitiva, son los comportamientos, actitudes y valores sociales establecidos para cada uno de los géneros.

Una manera más específica y vivencial, para referirnos a los roles de género, podemos decir que éstos determinan acciones, vislumbran las expectativas y normas que una sociedad establece en la actuación y sentir de una persona en función de que sea mujer o hombre, atribuyendo funciones que finalmente son asumidas diferencialmente por mujeres y hombres.

Puede decirse entonces, que los roles femeninos son los relacionados con todas las tareas asociadas a la reproducción, crianza, cuidados, sustento emocional, protección, etc. e inscritos fundamentalmente al ámbito doméstico (ámbito privado). En tanto los roles masculinos están asociados a las tareas que tienen que ver con lo productivo, mantenimiento y sustento económico, principalmente desarrollados en el ámbito público, lo que además legitima el poder hegemónico.

En síntesis, los roles de género son aquellas actividades que socialmente se escogen para cada individuo dependiendo de su sexo, lo que viene a promover la 
diferencia de tareas de acuerdo a si se es hombre o mujer, y juegan un papel de cuantiosa importancia, porque como se mencionó anteriormente, van a legitimar esas ideas, creencias atribuidas a hombres o mujeres, que generalmente invisibilizan la verdadera perspectiva del ser humano integral, que finalmente los llevan a crear esa luchas de poder en detrimento de una posible consolidación y comprensión para el logro de esa necesaria equidad.

Ahora bien relacionado a lo anterior, es que se dejo de último la conceptualización de género en sí y sus teorías, esto porque implican precisamente el análisis de los factores históricos y psicosociales que llevaron a las luchas existentes desde siglos atrás y hasta el momento actual, el género constituye la categoría de la construcción social y simbólica de hombres y mujeres sobre la base de la diferencia sexual.

De esta manera, cuando se revisan las definiciones de género, aparece la acostumbrada definición que estipula el conjunto de atributos, de atribuciones, de características asignadas al sexo, que con su valioso análisis Lagarde (1990), aclara que ese conjunto de características que tradicionalmente se pensaban como de origen sexual, en realidad son históricas, por eso las características son "atribuidas", lo que haría, que se repita el mito perteneciente al sentido común, de la ideología dominante, a las religiones, etc. el cual todo lo que somos las mujeres y los hombres es "natural" y tiene un origen "sexual", mito que tiene un contexto social e histórico, y hay que tener claro que es un "mito", por lo tanto que sobre este fueron educadas la mayoría de las personas.

De acuerdo a la palabra mito que tiene su origen del griego mythos ("cuento"), que describe a un relato de hechos maravillosos cuyos protagonistas son personajes sobrenaturales (dioses, monstruos), extraordinarios (héroes), por lo que puede desprenderse de lo anterior que el género desde lo sexual, se puede conceptualizar dentro de lo irreal.

Debido a ese proceso histórico lleno de atribuciones, mitos, intereses de ideologías dominantes, es que Lagarde (1990), manifiesta que finalmente siendo el conocimiento profundamente transformador, nos ha cambiado de manera muy diferente y que lo que hay es un fenómeno cultural, un proceso deconstructivo de género, que abarca a la identidad, la afectividad y la subjetividad.

Por otro lado Gúzman y Pérez en 2007, hacen una exhaustiva revisión sobre las teorías de género, para concluir que es importante determinar si es posible hablar de teorías feministas o teorías de género o si está por crearse una teoría científica de género, que basándose en alguna de las teorías feministas que existen, tenga recursos epistémicos, metodológicos y lógicos consistentes para ser llamada científica, y cuyo objeto de estudio más que la desigualdad, la inequidad o la opresión, pueda descubrir, explicar, y comprender las relaciones sociales entre los géneros que abarcan más que la simple dicotomía masculinofemenino en una sociedad abierta y compleja, en la que siempre han existido 
relaciones masculino-masculino, femenino-femenino, y sus múltiples variaciones que implican un análisis que las actuales teorías no alcanzan a comprender.

Un aspecto de relevancia, que se analiza también en este artículo a través de la encuesta aplicada, es la utilización de algunos refranes, porque es importante conocer y descifrar los mensajes semánticos, culturales, ideológicos que están instaurados la mayor parte de las veces de manera inconsciente en las formas en que se hace su reproducción social, como parte de esa legitimación, mantenimiento y conservación de las relaciones de género asimétricas entre hombres y mujeres. A través de la utilización de esos mensajes como lo son los refranes, se encuentra encubierta la violencia, la discriminación, propios de las prácticas y comportamientos sociales aceptados, e integrados al lenguaje cotidiano de un colectivo social, siendo aceptados o asimilados como algo chistoso o gracioso.

Para Fernández (2002), la visión dominante o hegemónica de la división sexual se expresa en discursos sociales y narrativas culturales como los refranes, y considera a los refranes como "un género del habla" más que una forma de lenguaje, que pasan a través del tiempo transmitiendo su mensaje original.

Además menciona la autora anterior, que el control ejercido sobre el uso del lenguaje y el potencial semántico y expresivo de la lengua, es una forma de control de los sectores dominantes, en general, y del grupo masculino, en particular. Así considera ella, que la violencia simbólica a través de la expresión verbal es parte de la dinámica de mantenimiento de la desigualdad social, étnica, de edad y de género, dentro del campo de la cultura popular, de la producción simbólica y de la comunicación en general. Fernández (2002).

En este artículo y con la utilización de varios refranes en la encuesta aplicada, se hace un análisis que permite observar esa violencia cotidiana utilizada de forma oculta e inconsciente de expresiones verbales que se instauran e introyectan como naturales y se aceptan sin cuestionamientos, sin medir sus consecuencias. Precisamente, los estereotipos y refranes, están llenos de significados metafóricos y tanto hombres como mujeres los utilizan en la lingüística de su diario comportamiento emocional y cognitivo.

Puede observarse, que el recorrido hecho en la revisión de los distintos conceptos implicados en este estudio derivados de las teorías de género, no ha sido fácil ni ha finalizado, y tiene diversas aportaciones de distintos enfoques interdisciplinarios, pero tampoco es necesario que finalice con una solución, porque el conocimiento científico de todas maneras es dinámico, pues como afirma Kuhn (1971) "ninguna teoría resuelve nunca todos los problemas a que en un momento dado se enfrenta, ni es frecuente que las soluciones ya alcanzadas sean perfectas. Al contrario, es justamente lo incompleto y lo imperfecto del ajuste entre la teoría y los datos existentes lo que, en cualquier momento, define muchos de los enigmas que caracterizan a la ciencia normal".

Los cambios o transformaciones que le esperan a la temática de género seguirán un recorrido a largo plazo, como lo es el cambio de actitudes, 
comportamientos, patrones sociales, etc., aspectos que fácilmente pueden evidenciarse en el diario vivir, lo importante es que se encaminan aunque sea paso a paso. Como ejemplo de esto y para cerrar este apartado, en el Semanario Universidad de la última semana de marzo del 2013, en uno de sus titulares se enuncia "Estereotipo de género prevalece en algunas carreras universitarias", y se afirma que con datos de los estudiantes admitidos a la Universidad de Costa Rica entre 2009 y 2013, siguen existiendo carreras femeninas y carreras masculinas. Así carreras como administración aduanera, trabajo social, psicología, educación preescolar y enseñanza del inglés, tienen mayor población femenina, mientras ingeniería eléctrica y civil, agronomía, computación e informática están integradas predominantemente por hombres. Se concluye que las carreras de ingeniería eléctrica y computación e informática en promedio nueve de cada diez estudiantes, son hombres, en tanto en educación preescolar nueve de cada diez en promedio son mujeres y en trabajo social ocho de cada diez estudiantes son mujeres.

Como corroboración de lo anterior, en una investigación de Barberá, Candela y Ramos en 2008, señalan como hipótesis inicial que en las profesiones de Psicología e Ingeniería Industrial, la percepción de los estudiantes sigue siendo estereotipada, de modo que Psicología se considera una carrera femenina en la medida en que entre sus estudiantes hay muchas mujeres y, además, la profesión se vincula con actividades de ayuda y cuidado, que se perciben socialmente como tareas propias de mujeres, en tanto Ingeniería es percibida como una carrera de hombres. Lo anterior indica que lo que sucede en la Universidad de Costa Rica, no es ajeno a universidades como la de Valencia en España, donde los autores Ilevaron a cabo su investigación, y aunque los resultados de la encuesta aplicada para el relato del presente artículo, son muy halagüeños, según la investigación anterior y los datos del Semanario Universidad evidencian comportamientos estereotipados en lo que se refiere en este caso a la elección de carreras universitarias.

La anterior cita, sirve para darnos cuenta que las transformaciones van a paso lento, se evidencian aún las dificultades para cambiar los roles de género asignados socialmente desde nuestros ancestros, en donde por siglos se ha asumido que hombres y mujeres tienen capacidades distintas, y lastimosamente son dadas de forma inequitativa, con desventajas de unos sobre los otros, aún así queda la esperanza y la ilusión de un mundo con equidad e igualdad.

\section{Metodología}

\section{A. Estrategia}

Se propuso un estudio descriptivo con estudiantes de la Sede Rodrigo Facio de la Universidad de Costa Rica, a través de la aplicación de una encuesta para 
determinar la vigencia de algunos estereotipos, roles y refranes relacionados con la temática del género.

\section{B. Procedimiento de muestreo}

Para la selección de los y las informantes, se estableció una escogencia al azar dentro del campus universitario de la Sede Rodrigo Facio de la UCR, en donde se aplicó la encuesta a todos y todas las estudiantes que tuvieran la disposición de responderla de forma auto-administrada, ya fuese en los pasillos, en el pretil, en aulas, o en cualquiera de los espacios posibles, y dicha encuesta además fue anónima.

Se trabajó con una muestra total 505 estudiantes, lo cual permitió establecer generalizaciones a la población con un nivel de error máximo del $5 \%$ y un nivel de confianza del 95\%.

A continuación se detallan las principales características de las personas encuestadas.

Tabla 1. Distribución de frecuencias según sexo

\begin{tabular}{lll}
\hline \multirow{2}{*}{ Sexo } & \multicolumn{2}{l}{ Frecuencias } \\
\cline { 2 - 3 } & Absoluta & Relativa \\
\hline Masculino & 203 & $40 \%$ \\
\hline Femenino & 302 & $60 \%$ \\
\hline TOTAL & 505 & $100 \%$ \\
\hline
\end{tabular}

Tabla 2. Distribución de frecuencias según edad

\begin{tabular}{lll}
\hline \multirow{2}{*}{ Edad } & \multicolumn{2}{l}{ Frecuencias } \\
\cline { 2 - 3 } & Absoluta & Relativa \\
\hline $17-26$ & 480 & $95.0 \%$ \\
\hline $27-36$ & 18 & $3.6 \%$ \\
\hline $37-46$ & 3 & $0.6 \%$ \\
\hline $47-56$ & 2 & $0.4 \%$ \\
\hline NR & 2 & $0.4 \%$ \\
\hline
\end{tabular}


Tabla 3. Distribución de la muestra según lugar de procedencia

\begin{tabular}{ll}
\hline San José & 250 \\
\hline Alajuela & 78 \\
\hline Cartago & 60 \\
\hline Heredia & 66 \\
\hline Guanacaste & 9 \\
\hline Puntarenas & 15 \\
\hline Limón & 10 \\
\hline NR & 16 \\
\hline Otros & 1 \\
\hline TOTAL & 505 \\
\hline
\end{tabular}

Tabla 4. Distribución de la muestra según áreas de carreras de estudio

\begin{tabular}{ll}
\hline Ciencias Sociales & 293 \\
\hline Ciencias Agroalimentarias & 10 \\
\hline Ingenierías & 70 \\
\hline Artes y Letras & 39 \\
\hline Salud & 71 \\
\hline Ciencias Básicas & 21 \\
\hline NR & 1 \\
\hline TOTAL & 505 \\
\hline
\end{tabular}

\section{Instrumento de recolección de la información}

Se utilizó un cuestionario estructurado para la recolección de la información, diseñado expresamente para este estudio por la investigadora. El cuestionario de encuesta empleado incluye 24 ítems: 5 preguntas para recolectar la información socio-demográfica necesaria (carrera, número de carnet universitario, edad, lugar de procedencia y sexo). Las siguientes 16 preguntas de la herramienta buscan identificar los estereotipos y roles de género aún persistentes entre la población universitaria encuestada, teniendo claro que dichos estudiantes pueden ser originarios de cualquier parte del país. Finalmente se incorporan 3 refranes relacionados con género, utilizados durante muchas décadas. 


\section{Recolección de la información}

La encuesta fue aplicada con la colaboración de los y las estudiantes del curso de Psicología General, quienes apoyaron a los y las encuestados/as con terminología y otros.

Además aunque los y las encuestados/as fueron seleccionados/as al azar, si se realizó un esfuerzo consciente para que esta muestra contara con estudiantes de diversas carreras, con la idea de lograr una muestra lo más representativa posible. El instrumento se aplicó de forma auto administrada con el fin de garantizar mayor confidencialidad en las respuestas. Asimismo, para este mismo fin, el cuestionario fue anónimo, con el objetivo de garantizar respuestas de mayor sinceridad.

\section{E. Procedimiento de análisis}

Los cuestionarios se procesaron electrónicamente a través del programa Excel, con el fin de obtener distribuciones de frecuencia para cada uno de los ítems de la encuesta. Debe recordarse que, por la metodología de recolección utilizada, es previsible un número pequeño de no-respuestas y en consecuencia, el tamaño de la muestra puede variar.

En el capítulo de resultados se muestran solo aquellos contrastes que resultaron estadísticamente significativos con un a menor o igual a 0,05.

\section{Análisis de Resultados de la Encuesta}

Para facilitar la lectura y comprensión de los resultados, se explican estos en porcentajes, y por el tamaño de este artículo se omiten los cuadros y los gráficos. A continuación se hace una descripción de los resultados por cada pregunta y respuesta, para finalizar con la conclusión de resultados.

Se describen las preguntas y sus respuestas en porcentajes, dadas por hombres y mujeres participantes, la edad, lugar de procedencia y carrera no mostraron diferencias significativas, ni tampoco el sexo, sin embargo hay algunas respuestas interesantes respecto al sexo de los estudiantes.

Pregunta 1: ¿Cuando una pareja sale, para usted lo lógico es que los gastos los cubra? Hombre Mujer Ambos

Respuesta: El $85 \%$ de la muestra respondió que ambos. De la población masculina el 82 \% respondió también que ambos deberían cubrir los gastos. Lo que indica que el estereotipo de que el hombre debería ser quién cubre los gastos de una salida parece no estar vigente en la población encuestada, pero no puede omitirse que en la modificación de los estereotipos, destacan tanto los 
factores cognitivos cómo los factores sociales, y en el caso de la muestra de esta investigación, hay que tener en cuenta que son personas con alta instrucción académica, lo que ha ayudado no solo en la cognición, sino que en lo social posiblemente las relaciones también sean contextualizadas al ámbito universitario.

Pregunta 2: ¿Para usted quién debe pedir una relación formal de noviazgo? El hombre La mujer Cualquiera de los dos

Respuesta: Un 59\% de la muestra respondió que cualquiera puede pedir una relación formal de noviazgo. De la muestra masculina un $75 \%$ opina que cualquiera puede pedir la relación, lo cual indica que en la mayoría de la población el estereotipo de que debía ser el hombre el que declarará una relación de noviazgo parece no ser real en esta muestra.

Pregunta 3: ¿Cree usted que los hombres deben responsabilizarse en mayor medida que las mujeres del ingreso familiar?

Respuesta: Un 85\% respondió que No y de la muestra total, la población masculina respondió en un $78 \%$ que esto no es solamente responsabilidad de los hombres.

Pregunta 4: ¿Opina que la manutención económica que por ley el hombre debe dar a la mujer cuando se divorcia, es una medida injusta, ya que mientras el marido ha trabajado, la esposa se la ha pasado en casa?

Respuesta: Los encuestados en un $71 \%$ considera que es injusta, de esta muestra total un $62 \%$ de los hombres la consideran injusta y un $77 \%$ de las mujeres también la considera injusta. Llama la atención que una minoría entre ellas mujeres consideren que es justo que los hombres asuman la manutención.

Pregunta 5: ¿Considera usted que las y los hijos de una madre que trabaja fuera de la casa pueden crecer tan sanos y felices como los de una madre que está en la casa?

Respuesta: El $70 \%$ de la muestra total considera que los hijos e hijas de una madre que trabaja fuera de la casa pueden crecer tan sanos como los de una madre que no trabaja fuera de la casa. La población masculina en un $60 \%$ considero que si es igual. Esta respuesta positiva, sobre todo ahora que la mujer se desempeña en espacios públicos y que tal vez por años se cargó de culpa al asumir roles que no eran los tradicionales.

Pregunta 6: ¿Cree usted que las cremas de cara o cuerpo, la depilación, el pedicura o manicure son para? Hombres, Mujeres o Ambos.

Respuesta: Un $81 \%$ opino que los cuidados estéticos son iguales para hombres que para mujeres. De la muestra masculina la mayoría el $77 \%$ opina que 
si son para ambos, indicando que se ha dado un cambio de actitud en el esquema de la belleza o estética, la cual por siglos fue atribuida a mujeres.

Pregunta 7: Cuando a usted lo atienden en una clínica u hospital porque está enfermo ¿quién cree lo atiende mejor? Hombre, Mujer o No importa

Respuesta: Un $87 \%$ considera que cualquiera puede atenderlo bien, que no importa si es hombre o mujer. Puede deducirse que no hay mayor cuestionamiento y por lo tanto no hay estereotipo.

Pregunta 8: ¿Cree usted que es mejor una relación amorosa entre? Un hombre mayor y una mujer joven, Una mujer mayor y un hombre joven, Ambas, Ninguna.

Respuesta: Un 56\% consideró que cualquiera de las relaciones puede ser buena, sin embargo, al analizar las respuestas en cada uno de los grupos de forma independiente, se observa que tanto para hombres como para mujeres la consideración mayoritaria es que una relación puede ser positiva para cualquiera independientemente de la edad, no obstante, la comparación entre los dos grupos resulta muy interesante, del porcentaje que dijo que era mejor una relación entre un hombre mayor y una mujer joven, de esa cantidad minoritaria (22\%), un $25 \%$ es masculina (28 respuestas absolutas) y el $75 \%$ femenina ( 83 respuestas absolutas).

Puede señalarse, que la opinión de una relación amorosa entre un hombre mayor y una mujer joven sigue siendo más aceptada por hombres como por mujeres, asociándose una respuesta ciertamente residual de una relación por siglos aceptada, y por lo tanto puede decirse que en menor medida siempre se mantiene el estereotipo de percibir de forma más tangible la relación entre un hombre mayor con una mujer joven, se podría señalar de forma especulativa que de la población universitaria encuestada una cantidad de mujeres legitiman hoy en dúa algunos de los estereotipos machistas. Pese a los cambios en el comportamiento y en la actitud hacia la posición de hombres y de mujeres que pueden experimentar las personas, es innegable que el sistema ideológico que fundamenta lo social presenta obstáculos para el cambio, pues es parte de una cultura de visión antagónica entre los sexos.

\section{Pregunta 9: ¿Cuando sale de paseo con su familia quién es el que generalmente conduce? Padre, Madre, Un hijo, Una hija, Otro.}

Respuesta: Un 67.7\% manifestó que conduce el padre, indicando que el estereotipo de conducción por parte de los hombres cuando se sale en familia se mantiene. Es interesante que aún hoy en día que hay mucha población femenina circulando con sus vehículos en las calles, pero precisamente cuando se trata 
de tomar el volante en el ámbito familiar, es el hombre el que conduce. Por supuesto, como todos participamos activamente en la culturización, organizando cognitivamente las percepciones relativas al rol de sexo a partir de nuestras nociones sobre el mundo, así adquirimos la constancia de género ((los hombres juegan al fútbol, papá es el que conduce, sale a trabajar, las mujeres cuidan de los hijos e hijas, van al gimnasio, hacen manualidades) asumiéndose como algo natural, aunque en la actualidad la mujer haga todos esos roles y más, ni siquiera se cuestiona por qué el padre se sienta al volante en el auto de la madre.

Lo mencionado anteriormente puede que se relacione con algo tan introyectado en la psique humana como lo son, los modelos adoptados por uno u otro sexo, donde los modelos masculinos son valorados más positivamente que los femeninos, porque implican poder, habilidades, independencia, etc.

\section{Pregunta 10: ¿Cuando una pareja decide no tener más hijos, quién debería someterse a una cirugía? Hombre, Mujer, Cualquiera de los dos, Otro.}

Respuesta: El 81\% consideró que cualquiera de los dos puede operarse si no quieren tener más hijos. Puede que esto evidencie la ruptura o no aceptación de estereotipos, que permiten un cambio de actitudes, favorables para todos los seres humanos en pro de esa igualdad por la que se dan grandes luchas en la actualidad. No obstante lo anterior, la instrucción académica parece proteger a hombres y a mujeres, de ser catalogados en un esquema estereotipado, ya que la preparación parece estar ayudando a la ruptura de ese conjunto de prejuicios, creencias y actitudes erróneas.

\section{Pregunta 11: ¿Quién cree usted expresa más los sentimientos? Los hombres, Las mujeres, Ambos.}

Respuesta: Un 61\% de la muestra opina que expresan más los sentimientos las mujeres, y de ésta población masculina en igual porcentaje opina que las mujeres.

No debe olvidarse que el estereotipo es ante todo emocional, por lo que si se pretende desde consideraciones intelectuales cambiarlo, no hay mucho que hacer. Estos son transmitidos de generación en generación, y conllevan un cambio pausado en su progreso, por lo que no coinciden con la realidad. Así por ejemplo, de acuerdo a las respuestas de la encuesta aplicada, puede observarse que el contenido tradicional de los estereotipos de género que enmarcan el comportamiento de los hombres como agresivos, estables emocionalmente, autocontrolados, dominantes, con un yo fuerte, con grandes aptitudes intelectuales y sobre todo poco afectivos, por lo que se les considera más racionales, sigue vigente. En tanto a las mujeres, por su parte, se les considera sumisas, inestables emocionalmente, tiernas, poco controladas, dependientes, poco intelectuales pero 
intuitivas e irracionales, y muy sentimentales, aspecto que se evidencia en la percepción de los y las encuestadas, que creen que las mujeres expresan más lo sentimientos que los hombres.

Dado lo anterior, puede señalarse que una vez más los estereotipos logran su cometido de reflejar que las creencias, los roles, rasgos, características o atributos que distinguen a hombres y mujeres, como lo menciona Parga (2008), fijando socialmente ese modelo de lo que tienen que ser o desempeñar cada uno desde cada rol, con lo que se establecen las relaciones de desigualdad, que finalmente deforman la realidad.

Pregunta 12: ¿Piensa usted que la mayor parte de personas ebrias son? Hombres, Mujeres o Ambos.

Respuesta: El 56\% consideró que ambos pueden ser ebrios, sin embargo un $41 \%$ piensa que los hombres, y solo un $2 \%$ opinó que las mujeres. Aunque en minoría, siempre se percibe al hombre como mayor consumidor de licor, lo que no deja de ser real, pues en Costa Rica, de acuerdo a datos del año 2011, de la Sección de Estadística, Departamento de Planificación del Poder Judicial, la mayoría de accidentes automovilísticos se dan por hombres con altos niveles etílicos en su sangre, representando los hombres el 80 por cierto de las muertes.

\section{Pregunta 13: ¿Considera que cortar el zacate o césped en el jardín de la casa es una tarea de? Padre, Madre, Hijos, Todos.}

Respuesta: El 77\% considera que es una tarea de todos. Como puede observarse el estereotipo se ha eliminado, por años era una tarea o rol que se le asignaba a los hombres de la casa, y ahora parece ser una tarea compartida por todos los miembros de la familia.

\section{Pregunta 14: ¿Usted cree que son mejores choferes? Los hombres, Las mujeres o Ambos.}

Respuesta: El 65\% consideró que ambos son buenos choferes, sin embargo de la muestra masculina el $42 \%$ opinó que los hombres son mejores choferes. Es evidente que quedan vestigios de ese estereotipo hasta el momento actual, estereotipo que de alguna manera mantiene esos comportamientos viriles llenos de poder, aún cuando las estadísticas demuestran que los hombres provocan más accidentes en las carreteras que las mujeres, que son más agresivos y temerarios.

Pregunta 15: ¿Cuando se divorcia una pareja, es importante que los hijos queden al cuidado de: Padre, Madre, o se debe compartir el cuido?

Respuesta: Un 92\% considera que el cuido debe ser compartido. La respuesta indica otra actitud positiva que elimina otro estereotipo, en el que se pensaba que en caso de un divorcio los hijos debían ser asumidos por la madre. 
Es interesante y positivo, ver en las calles o en las redes sociales, el rol de padres que asumen actualmente los hombres jóvenes.

Pregunta 16: ¿Los trabajos del campo como la agricultura los hacen mejor? Los hombres, Las mujeres, o Ambos.

Respuesta: Un 74.3\% opina que ambos pueden hacer los trabajos de campo bien. El 25\% restante considera que los hombres lo hacen mejor. Se esperaría una respuesta mayoritariamente hacia los hombres, porque aún en la actualidad, este tipo de tarea se evidencia en las zonas donde se trabaja en agricultura la contratación de hombres, pero puede que el planteamiento de la pregunta no sea adecuado o exista la necesidad de brindar una impresión de jóvenes sin estereotipos, pero podría especularse o plantearse de otra forma la pregunta en una futura investigación.

\section{Refrán N¹: "Es mejor ser deseada que sobrada"}

Respuesta: El 90\% si lo ha escuchado, lo que indica que se mantiene vigente.

\section{Refrán N²: "Los hombres Ilegan hasta donde la mujer lo permita"}

Respuesta: El 90\% de la muestra lo ha escuchado, es decir, que este refrán se mantiene vigente.

\section{Refrán N03: "Jalan más un par de tetas que una yunta de bueyes"}

Respuesta: Este refrán solo el $60 \%$ lo ha escuchado y siendo mayoría se mantiene vigente.

Para finalizar, es indudable de acuerdo a las respuestas a los refranes que estos siguen manteniendo su vigencia, pues son conocidos por la mayoría de los y las jóvenes en un porcentaje bastante alto, con el inconveniente de que estos han sido relatados por décadas de décadas, y como se menciono en el marco teórico de este artículo, a través de la utilización de los refranes, se encuentra encubierta la violencia, la discriminación, propios de las prácticas y comportamientos sociales aceptados, asimilados como algo chistoso o gracioso, siendo perjudiciales para ambos sexos.

\section{Conclusiones}

A través del análisis de los resultados fue posible identificar que la mayoría de roles y estereotipos presentan disposiciones parecidas en sus respuestas, lo cual permite comprender que parece estar desapareciendo la tendencia a la utilización de los roles y los estereotipos tradicionales que se daban entre hombres y mujeres. Lo anterior, muestra que los estereotipos son sensibles al paso del tiempo, además que la transmisión y asimilación de los mismos es compleja, aunado a esto puede verse con los resultados que se está generando un cambio 
en la dinámica social actual, por lo menos respecto a la población universitaria participante en esta investigación.

Sobre todo dichos resultados refieren a cambios en los estereotipos relacionados con el sexo, porque estos se asocian a roles, y aunque pueden ser positivos o negativos, siempre se van de la mano con actitudes prejuiciosas y discriminatorias. Sobre esto según Diekman, Goodfriend y Goodwin en 2004, lograron demostrar que los observadores percibían que las mujeres habían ganado poder político, económico, laboral, individual y relacional del pasado al futuro. Y además consideraban que las mujeres más que los hombres tendrían más poder en un futuro, lo que evidencia que se están realizando cambios importantes de forma pausada en el tiempo.

Los resultados señalan que se está dando un cambio en la percepción de los roles de género, que históricamente habían sido atribuidos solo a uno de los sexos, y que hoy se ven como propios de cualquiera de los dos sexos, tal es el caso de los estereotipos de cuidados personales, estos cuidados años atrás eran asignados solo a las mujeres y en la encuesta aplicada la mayoría los consideró necesarios para mujeres como para hombres.

La responsabilidad de la carga económica, que antiguamente se asumía para los hombres, logra un cambio en esta muestra, ya que la mayoría de los participantes opina que debe ser asumida tanto por hombres como por mujeres, y también se modifica el rol de la toma de decisiones en una relación amorosa para solicitar una relación formal, pues consideraron la mayoría que esta decisión es tanto de mujeres como de hombres.

Otro rol que se percibe diferente en la actualidad, es el de la mujer que trabaja fuera de la casa y lo que esto pueda afectar a los hijos e hijas, pues la mayoría consideró que no tiene por qué perjudicar la felicidad y crecimiento adecuado de los hijos e hijas el hecho de que la madre labore fuera del hogar, asimismo opinaron que hombres y mujeres deben compartir el cuidado de los niños y niñas. De esta manera puede verse con los resultados que la evolución o dinámica de las transformaciones sociales, los roles y estereotipos que ocupan mujeres y hombres, no solo se relacionan y sino que se han modificado los estereotipos de género con los distintos tipos de poder.

Las características de género vinculadas a hombres y mujeres han cambiado a lo largo del tiempo, ahora ambos comparten el poder que, tradicionalmente correspondía solo al género masculino, lo que indudablemente ha transformado la percepción sobre los estereotipos de género como lo demuestran los resultados de la encuesta aplicada a la población universitaria.

Un rol que parece estar manteniendo su vigencia es el de hombre como chofer los fines de semana en los paseos familiares, donde la mayoría mencionó que el padre es el que conduce en los paseos familiares, aunque no hay investigaciones del número de mujeres y hombres conductores en Costa Rica, 
según estadísticas del año 2011, de la Sección de Estadística, Departamento de Planificación del Poder Judicial, demuestran que los hombres conducen más agresivo, que provocan más accidentes que las mujeres, y que un $80 \%$ de las muertes en accidentes son hombres.

El estereotipo de que era la mujer la que debía acudir a una esterilización cuando se tomaba la decisión de no tener más hijos o hijas, también dio un giro, ya que la mayoría considera que cualquiera puede hacerse dicha cirugía o cualquier proceso que le permita no tener hijos o hijas si así lo desea.

Por otra parte, aunque en porcentaje no fue tan diferente, si fue significativo la percepción de la mujer como la más sentimental en la expresión de sentimientos, característica adjudicada en función del sexo, lo que evidencia que sigue presente en el inconsciente colectivo la percepción de la mujer como la dulce, la tierna, la cariñosa, etc., lo que indica que se llevará todavía algún tiempo cambiar esta percepción.

Respecto a los resultados de las respuestas de los tres refranes, siguen siendo escuchados por la mayoría de encuestados, llamando la atención que a pesar de que en toda la encuesta se observó un cambio de actitudes en relación a los estereotipos, en las respuestas sobre los refranes se evidencia una mayor vigencia de una construcción semántica que conlleva en sí estereotipos de forma menos perceptible, como se menciona en este artículo. Así, las funciones constructivas de los estereotipos que son esquemas ampliamente compartidos en el mundo social, son preconstruidos, contribuyen a organizar las representaciones colectivas, las opiniones intercambiadas y el imaginario social, además adoptan una forma verbal fija para comunicar un mensaje abstracto o conceptual global entre los que están los refranes, siendo recurrentes y repetidos en múltiples situaciones. Así los refranes expresan de acuerdo a Poncela (2001), la visión hegemónica de la división sexual con la consecuente función de transmitir a través del tiempo su mensaje original, mensaje que en primera instancia forma parte del control de sectores dominantes, como el sector masculino, en particular. Lo anterior coadyuva al mantenimiento de la dinámica de desigualdad, sin excluir la de género.

Finalmente, puede verse de acuerdo a las respuestas, que en la actualidad en una población como la universitaria, se mantienen todavía sutilmente formas de poder que obedecen a los intereses ideológicos, no obstante, también se observan cambios del papel del hombre y de la mujer, que posiblemente se obedezcan a varios factores como lo es la incorporación de la mujer a la esfera pública desde lo laboral, lo que como consecuencia genera incertidumbre en los hombres en relación al poder que siempre han tenido, pues ya no son los únicos proveedores ni dueños del poder. Pero a la vez, se puede rescatar una percepción positiva de la valoración tanto de hombres como de mujeres, a través precisamente de un 
cambio en las apreciaciones de los estereotipos de acuerdo a los resultados, lo que puede estar generando la apertura hacia una sociedad más equitativa.

En términos generales se puede concluir entonces que los y las estudiantes universitarias de la Sede Rodrigo Facio de la UCR, han cambiado la percepción de los estereotipos en general, que la mayoría atribuye roles y estereotipos semejantes a ambos sexos, a pesar de que los refranes mantienen su vigencia. Por otra parte, sobre los resultados de esta investigación, es importante reflexionar si efectivamente las transformaciones sociales están teniendo un impacto en las relaciones de género, porque si bien es cierto hay cambios en la percepción de los estereotipos y roles de género, todavía algunos aspectos mantienen esas desigualdades. Sin embargo, para no opacar los resultados positivos dados por los encuestados y no desesperanzar en las posibilidades de una transformación, lo recomendable sería abrir paso a un reaprendizaje de todos los actores sociales, en el que las relaciones sociales, y las auto percepciones entre hombres y mujeres puedan generar una transformación con la idea de lograr a corto plazo esa equidad y tolerancia tan necesaria en la sociedad actual.

\section{Bibliografía}

Alfaro, S., Salazar C., y Cruz F., (2013 31 de marzo), Estereotipo de género prevalece en algunas carreras universitarias. Semanario Universidad. 2.

Alfonso P. y Aguado J. Estereotipos y coeducación, Consejo Comarcal del Bierzo, Recuperado en http://fongdcam.org/manuales/genero/datos/ docs/1 ARTICULOS $Y$ DOCUMENTOS DE REFERENCIA/D EDUCACION/ Estereotipos y Coeducacion.pdf

Allport, Gordon W. (1971). La naturaleza del prejuicio (4a Ed.). Eudeba: Buenos Aires. Argentina.

American Psychological Association. (2009). Publication manual of the American Psychological Association. (6th ed.) Washington, DC: American Psychological Association. Recuperado en: http://www.lib.uconn.edu/research/guides/apa. pdf

Barberá, E., Candela, C., y Ramos A. (2008). Elección de carrera, desarrollo profesional y estereotipos de género. Revista de Psicología Social, Universitat de València, España, 23 (2), 275-285

Cano, J. I. (1993). Los estereotipos sociales. El proceso de perpetuación a través de la memoria selectiva. (Tesis inédita de doctorado). Universidad Complutense de Madrid: Madrid, España. 
Chaves, A.L. (2005). Las relaciones de género en el contexto escolar. Un estudio de caso a nivel de educación preescolar. Recuperado en http://www.historia. fcs.ucr.ac.cr/articulos/esp-genero/4parte/CAP25Lupita.htm

Diekman, A. B., Goodfriend, W. \& Goodwin, S. (2004). Dynamic stereotypes of power: Perceived change and stability in gender hierarchies. Sex Roles, 50, 201-215. Recuperado en: Link.springer.com/article/10.1023/ B\%3ASERS.0000015552.22775.44

Fernández, A.M. (2002). Estereotipos y roles de género en el refranero popular. Charlatanas, mentirosas, malvadas y peligrosas. Proveedores, maltratados, machos y cornudos. (2a Ed.). Anthropos Editorial: Barcelona, España.

González, M. (2000). El sexismo en la Educación (1ª. Ed.). Ciudad Universitaria Rodrigo Facio, San José, Costa Rica: Editorial Universidad de Costa Rica.

Herrero, J. (junio, 2006). La teoría del estereotipo aplicada a un campo de la fraseología: las locuciones expresivas francesas y españolas. Revista de estudios literarios, $N^{\circ} 32$, Universidad Complutense de Madrid. Recuperado en http://pendientedemigracion.ucm.es/info/especulo/numero32/teoreste. $\underline{\mathrm{html}}$

Jung, Carl G. (1970). Arquetipos e Inconsciente Colectivo, Ediciones Paidós Ibérica, S.A.: Barcelona, España.

Kuhn, T.S. (1971). La estructura de las revoluciones científicas (1ª. Ed.). México, D.F., México: Fondo de Cultura Económica.

Lagarde, M. (1990). La multidimensionalidad de la categoría género y del feminismo. Recuperado en http://www.cubaenergia.cu/genero/teoria/t33.pdf

Parga, L. (2008). La construcción de los estereotipos del género femenino en la escuela secundaria (1a Ed.) México, Tlalpal. México: Editorial de la Universidad Pedagógica Nacional.

Poder Judicial (s.f.), Recuperado en www.poder-judicial.go.cr/planificacion/795PLA-2012.rtf

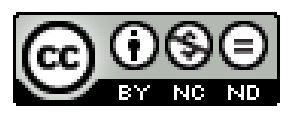

Este obra está bajo una licencia de Creative Commons ReconocimientoNoComercial-SinObraDerivada 4.0 Internacional. 